\title{
Variation of Electrical Resistivity with High Pressure in Ge-Te-Sn Glasses: A Composition Dependent Study
}

\author{
K.N.N. Prasad ${ }^{a, b}$, Chandasree Das ${ }^{c, \dagger}$, K. Rukmani ${ }^{a, *}$ And S. Asokan ${ }^{c}$ \\ ${ }^{a}$ Department of Physics, Bangalore University, Bangalore 560056, India \\ ${ }^{b}$ Department of Physics, BNM Institute of Technology, Bangalore 560070, India \\ ${ }^{c}$ Department of Instrumentation and Applied Physics, Indian Institute of Science, Bangalore 560012, India \\ (Received October 4, 2014; in final form February 11, 2015) \\ The variation of normalized electrical resistivity in the system of glasses $\mathrm{Ge}_{15} \mathrm{Te}_{85-x} \mathrm{Sn}_{x}$ with $(1 \leq x \leq 5)$ \\ has been studied as a function of high pressure for pressures up to $9.5 \mathrm{GPa}$. It is found that with the increase \\ in pressure, the resistivity decreases initially and shows an abrupt fall at a particular pressure, indicating the \\ phase transition from semiconductor to near metallic at these pressures, which lie in the range $1.5-2.5 \mathrm{GPa}$, and \\ then continues being metallic up to $9.5 \mathrm{GPa}$. This transition pressure is seen to decrease with the increase in the \\ percentage content of tin due to increasing metallicity of tin. The semiconductor to near metallic transition is \\ exactly reversible and may have its origin in a reduction of the band gap due to high pressure.
}

DOI: 10.12693 /APhysPolA.127.1666

PACS: $72.80 . \mathrm{Ng}$

\section{Introduction}

Chalcogenide glasses such as $\mathrm{Ge}_{2} \mathrm{Sb}_{2} \mathrm{Te}_{5}$ (GST) have been used successfully as non-volatile random access memories (NVRAMs) and this has generated renewed interest in these materials [1-6]. The successful use of these glasses as random access memories hinges on their ability to switch from a high resistance amorphous state to a low resistance crystalline one on the application of an appropriate electrical pulse [7]. Some of these glasses can be switched optically and the two states obtained can be differentiated by their different refractive indices. The search for better materials for these applications calls for improved understanding of the underlying phenomena and processes in these systems. High pressure is an important tool to probe the behavior of these materials and to gain insight into the processes taking place in these glasses.

Electrical resistivity measurements on glassy semiconductor under high pressure helps in understanding the pressure induced metallization or crystallization of these materials. In crystalline materials, the effect of pressure is to compress the unit cell and any phase transition that occurs is a cooperative phenomenon accompanied by a change in structure most of the times. The effect of pressure on glasses is more subtle, and hence of considerable interest. Chalcogenide glasses are generally semiconductors at room temperature and pressure, and undergo semiconductor to metal transition or crystallization when subjected to pressure [8-10]. The pressure induced crystallization and semiconductor to metal

\footnotetext{
${ }^{*}$ corresponding author; e-mail: rukmani99090yahoo.co.in

$\dagger$ Present address: Department of Electrical and Electronics Engineering, BMS College of Engineering, Bangalore 560018, India.
}

transition in amorphous semiconductors can be a continuous transformation or a discontinuous transition. Binary glasses such as $\mathrm{Ga}-\mathrm{Te}$ and $\mathrm{Al}-\mathrm{Te}$, which are of the type III-VI [11, 12] and As-Te which is of the type VVI [13] show continuous metallization under pressure. On the other hand, IV-VI chalcogenide glasses are found to exhibit sharp, discontinuous glassy semiconductor to crystalline metallic transition around 5-8 GPa [14, 15]. Further most V-VI chalcogenide glasses are usually found to remain a semiconductor even up to $10 \mathrm{GPa}$. Ternary glasses such as As-Te-Se [16], As-Te-Si [17] and AsTe-In [18] also show continuous metallization under high pressure.

Multi-component glasses such as Ge-Se-Bi [19], GeTe-As-S [20] exhibit continuous metallization whereas Tl-Ge-Se and Tl-Te-Ge glasses [21, 22] have been found to show discontinuous metallization. It can be seen from the above that chalcogenide glasses belonging to different groups show interesting difference in their pressure response, making high pressure studies on these materials an important tool in condensed matter physics.

The microscopic structure of chalcogenide glasses and the relation to their properties is of considerable interest in glass physics. Chalcogenide glasses are made up of various structural units such as tetrahedral, octahedral, bipyrimidal or linear depending on the local coordination around the element considered. Generally the $8-\mathrm{N}$ rule is applicable and the co-ordination of $\mathrm{Ge}=4$, $\mathrm{Te}=2$ and $\mathrm{Sn}=4$ in our sample. As the composition of a series of glasses is varied, the average co-ordination number changes and the glassy network moves through topological or rigidity thresholds. For average co-ordination numbers $\langle r\rangle$ less than 2.4 the glass is floppy and polymeric. At $\langle r\rangle=2.4$ which is the value at the rigidity percolation threshold (RPT), it is expected to change from floppy to rigid according to the theory of Thorpe. Generally compositions of the glasses studied are varied 
in such a way that the series spans the RPT so that the effect of the percolation thresholds can be studied.

The present work reports electrical resistivity measurements on bulk ternary $\mathrm{Ge}_{15} \mathrm{Te}_{85-x} \mathrm{Sn}_{x}$ glasses at high pressure as a function of the percentage content of tin and attempts to understand the effect of the addition of tin to the parent binary Ge-Te glass.

\section{Experimental procedure}

Bulk semiconducting glasses of a $\mathrm{Ge}_{15} \mathrm{Te}_{85-x} \mathrm{Sn}_{x}$ system with $1 \leq x \leq 5$ were synthesized by the conventional melt quenching technique. High purity $(99.999 \%)$ constituent elements of Ge, Te, and Sn in desired proportions were weighed with an accuracy of $\pm 0.1 \mathrm{mg}$ and were taken in flattened quartz ampoules of about $1 \mathrm{~mm}$ wall thickness. The constituents were evacuated to a vacuum better than $10^{-6}$ mbar and sealed. The sealed ampoules were loaded in a horizontal rotary furnace and were heated to $1000^{\circ} \mathrm{C}$ at the rate of about $100^{\circ} \mathrm{C} / \mathrm{h}$. The ampoules containing the melt were kept under continuous rotation for about $36 \mathrm{~h}$ at $10 \mathrm{rpm}$ to ensure homogenization and the ampoules were subsequently quenched in a bath of ice-water and $\mathrm{NaOH}$ mixture to obtain bulk glasses. The amorphous nature of the as-prepared samples was confirmed by X-ray diffraction (XRD) method.

The electrical resistivity measurements at high pressures up to $9.5 \mathrm{GPa}$ have been undertaken in a Bridgman opposed anvil cell, which works on the principle of massive support [23] with a working face diameter of $12 \mathrm{~mm}$ at room temperature. The design of the anvil is as reported in Ref. [24] and anvils are made of tungsten carbide and provided with binding rings made up of EN24 alloy steel. Pyrophyllite gaskets of $0.35 \mathrm{~mm}$ critical thickness were used in split gasket configuration. Steatite was used as the quasi-hydrostatic pressure-transmitting medium and in situ pressure calibration was achieved by using bismuth $(\mathrm{Bi})$ as the calibrant [25]. Samples of approximate dimension $2 \mathrm{~mm} \times 1.5 \mathrm{~mm} \times 0.4 \mathrm{~mm}$ were used for the present measurements of resistivity under pressure. A two-probe method was employed for the electrical measurements, a current of $50 \mathrm{nA}$ was passed through the sample using a constant current source and the voltage across sample was measured using a Keithley 614 nanovoltmeter. The error in the measurement of resistance was less than $\pm 0.06 \%$ at ambient pressure and the normalized resistivity avoids any errors arising from the measurement of the thickness of the sample. The error in the measurement of pressure was of the order of $\pm 2 \%$ or around $0.08 \mathrm{GPa}$. The samples were recovered from the pressure cell after being subjected to high pressures in some experiments. In these experiments, $\mathrm{NaCl}$ was used as a pressure transmitter and the sample after the high pressure cycle was collected by dissolving $\mathrm{NaCl}$ in distilled water. X-ray diffraction studies on the recovered samples were carried out to find the structure of the high pressure phase.

\section{Results}

Figure 1 shows the variation of normalized electrical resistivity $\left(\rho / \rho_{0}\right)$ with pressure in the samples
$\mathrm{Ge}_{15} \mathrm{Te}_{85-x} \mathrm{Sn}_{x}$ with $1 \leq x \leq 5$ for the entire series. Here $\rho_{0}$ is the resistivity at ambient pressure and is of the order of $10^{6} \Omega \mathrm{cm}$. The entire series of samples show a similar behavior under the application of pressure and it is evident from Fig. 1 that the normalized electrical resistivity decreases continuously with increase of pressure in the beginning and shows a sudden large abrupt decrease at a particular pressure (transition pressure $P_{\mathrm{T}}$ ) to near metallic values. Further increase in pressure beyond this results in a slow decrease in the resistivity values. The sudden fall in resistivity, indicates the presence of semiconductor to near metallic transition with the near metallic values being obtained around 1.5-2.5 GPa.

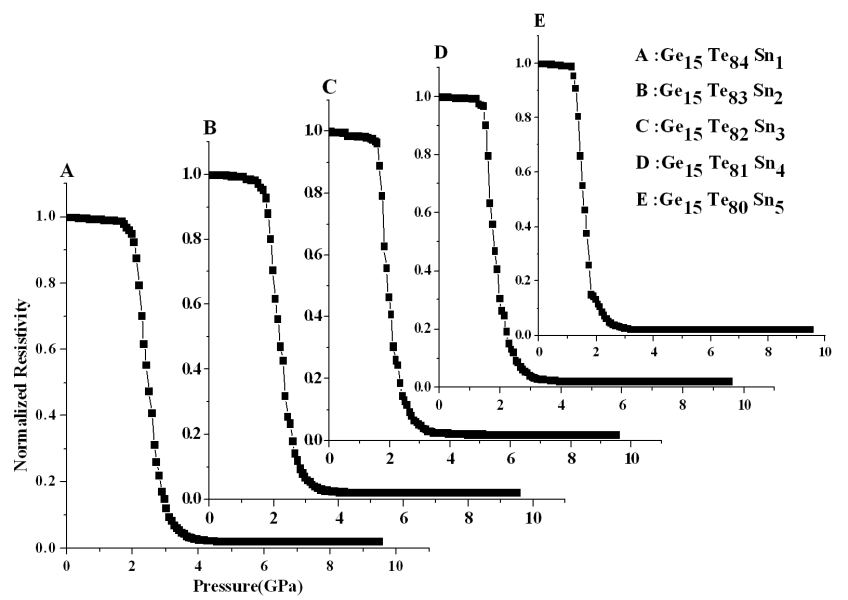

Fig. 1. Variation of the normalized electrical resistivity with pressure for $\mathrm{Ge}_{15} \mathrm{Te}_{84} \mathrm{Sn}_{1}, \mathrm{Ge}_{15} \mathrm{Te}_{83} \mathrm{Sn}_{2}$, $\mathrm{Ge}_{15} \mathrm{Te}_{82} \mathrm{Sn}_{3}, \mathrm{Ge}_{15} \mathrm{Te}_{81} \mathrm{Sn}_{4}$ and $\mathrm{Ge}_{15} \mathrm{Te}_{80} \mathrm{Sn}_{5}$ glasses.

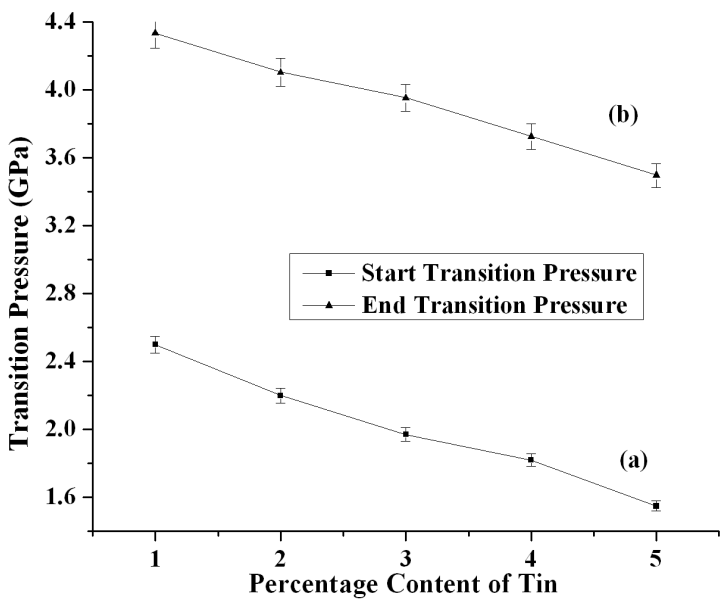

Fig. 2. (a) Variation of transition pressure with percentage content of Tin in $\mathrm{Ge}_{15} \mathrm{Te}_{85-x} \mathrm{Sn}_{x}$ glasses. The continuous line is a guide to the eye. (b) Variation of transition pressures (at which the transition is complete) with Percentage content of tin. The straight line is least square fit to the data points.

Figure 2 shows the variation of transition pressure as a function of percentage content of tin containing both the starting values as well as the ending values of the 
transition. The transition pressure is seen to decrease with increase in tin content. Figure $2 \mathrm{~b}$ shows the variation of pressures at which the transition is complete and near metallic values have been observed as a function of percentage content of tin.

Figure 3 shows the XRD pattern of representative samples recovered after applying pressure up to 9.5 GPa.

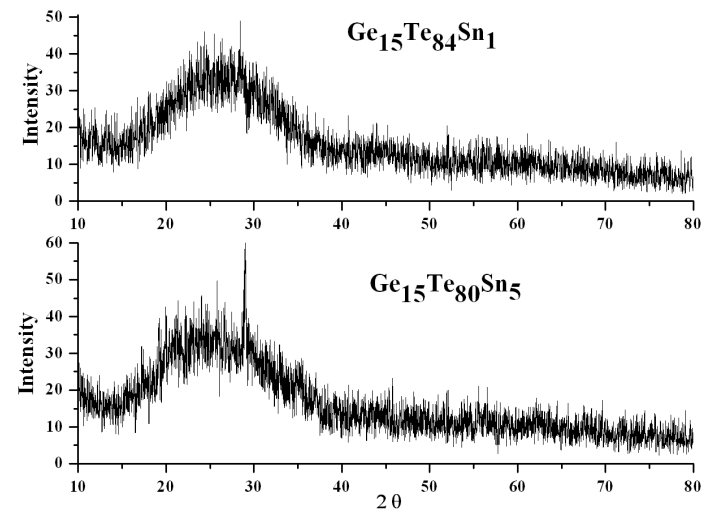

Fig. 3. XRD pattern of representative samples $\left(\mathrm{Sn}_{1}\right.$ and $\left.\mathrm{Sn}_{3}\right)$ recovered after pressurization.

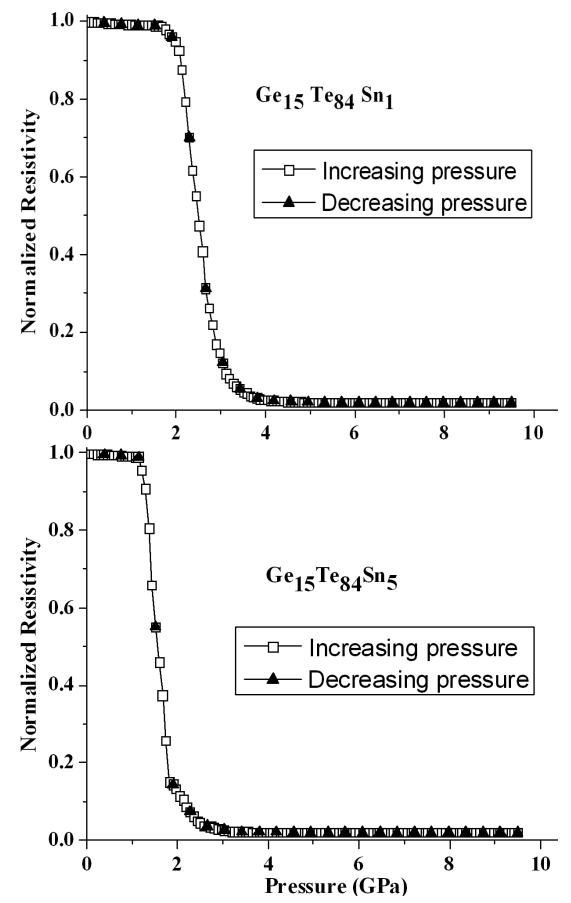

Fig. 4. Variation of normalized resistivity with pressure of representative samples $\left(\mathrm{Ge}_{15} \mathrm{Te}_{84} \mathrm{Sn}_{1}\right.$ \& $\mathrm{Ge}_{15} \mathrm{Te}_{84} \mathrm{Sn}_{5}$ ) when the pressure was decreased from 9.5 $\mathrm{GPa}$ to atmospheric pressure.

Figure 4 shows the variation of normalized resistivity as a function of pressure when the pressure was decreased from $8 \mathrm{GPa}$ to atmospheric pressure compared with the values for the compression cycle.

Figure 5 shows the variation of normalized resistivity with percentage content of tin at a constant pressure of $1.3 \mathrm{GPa}$.

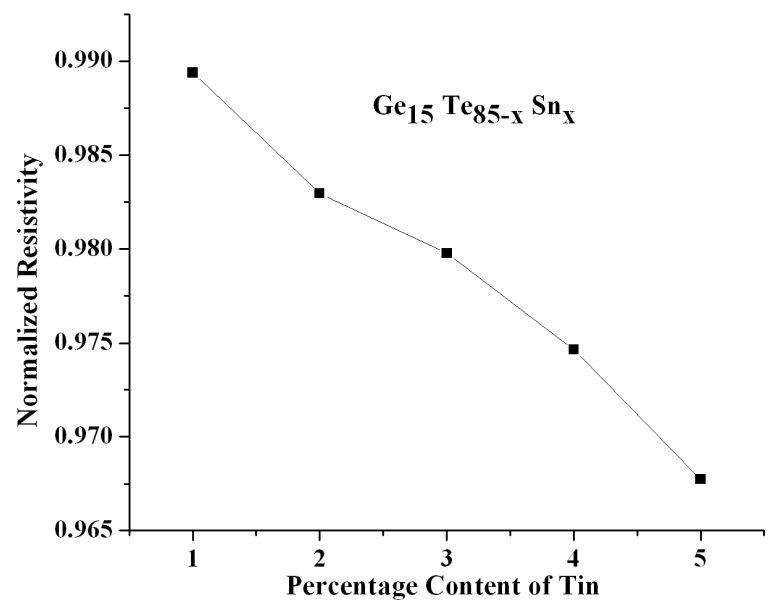

Fig. 5. Variation of normalized resistivity with percentage content of tin at a constant pressure of $1.3 \mathrm{GPa}$. The continuous line is a guide to the eye.

\section{Discussion}

The variation of normalized electrical resistivity with pressure of the samples in the entire series is shown in Fig. 1. Initially, the resistivity decreases with the increase in pressure, then shows a sudden drop to nearmetallic values around 1.5-2.5 GPa and then continues being near metallic up to $9.5 \mathrm{GPa}$. The near metallic high pressure phase has values of resistivity of the order of $10^{-2} \Omega \mathrm{cm}$. For the state to be called metallic, the resistivity has to fall below Mott's maximum metallic resistivity [26], and the above value is about an order of magnitude higher, and hence is termed near metallic. The parent binary glass to our present samples is $\mathrm{Ge}-\mathrm{Te}$ and these have been seen to undergo a sudden semiconductor to crystalline transition under the application of pressure [15]. On the addition of Sn atoms to this matrix, Sn atoms replace Te in the network and can form additional $\mathrm{Sn}-\mathrm{Te}, \mathrm{Sn}-\mathrm{Ge}$, and $\mathrm{Sn}-\mathrm{Sn}$ bonds. These tin atoms can bond with Te and increase the network connectivity as 4 coordinated tin is replacing 2 coordinated Te. If this were to happen, the system would move through percolation thresholds as the average coordination and the rigidity of the network increase in consonance with increasing Sn percentage. This increase in rigidity should contribute to an increase in the transition pressure with increase in $\mathrm{Sn}$ content. The transition pressure $P_{\mathrm{T}}$ is seen to decrease with increasing $\mathrm{Sn}$ concentration in the series of samples studied. Electrical switching as well as thermal stability [27] and other studies on this series of samples indicate that $\mathrm{Sn}$ (tin) atoms do not interact with the host matrix but form a phase separated network of their own and remain as inclusions in the parent matrix. Consequently, there is no enhancement of network connectivity and rigidity and this is reflected in the decrease in switching voltages with the addition of tin rather than an increase. The decrease in the switching voltages has been attributed to the increasing metallicity of tin as compared to Te. 
The decrease in $P_{\mathrm{T}}$, the transition pressure, with increase in Sn content (Fig. 2) is also due to the increasing metallicity of $\mathrm{Sn}$ as compared to the Te that it replaces. The effect of pressure on a glassy chalcogenide is to compress and shorten the bonds of the network in the glass. This decrease in interatomic distance increases the mobility of the electrons and reduces the bandgap. This results in increased conductivity or in other words a decrease in the resistivity at higher pressures. A sudden transition in the normalized resistivity to near metallic values can occur in two ways. One is because of a structural phase transition from amorphous to crystalline phase where the crystalline phase has very low resistivity. The second is due to a sudden closing of the band gap due to pressure with the conduction and valence bands meeting at some points. Various models have been proposed for the band structure of semiconducting glassy chalcogenides. The Marshall and Owen model [28] describes three types of states in these systems - non-localised states in the valence and conduction band, localized states at the band tails and localized defect states in the middle of the band gap near the Fermi energy. Increasing pressure reduces the band gap and metallization occurs when the gap between these three types of states is closed. In a network glass, if the rigidity increases, more pressure is needed to compress the system to the same point and produce the decrease in the energy gap. Hence the pressure needed to induce the transition to metallic state increases with increasing rigidity.

On the other hand, if tin is entering as an inclusion and is phase separated, these will introduce defect-like states in the gap and contribute to a decrease in the transition pressure. Since $\mathrm{Sn}$ is more metallic than Te, this will also contribute to a decrease in the transition pressure.

Both the beginning of the transition (Fig. 2a) and the end of it (Fig. 2b) are nearly straight lines pointing to a nearly inverse linear correlation between tin content and the transition pressure. A decrease in transition pressure with the additive (thallium) content was observed in $\mathrm{Ge}-\mathrm{Te}-\mathrm{Tl}$ glasses also [22] but the beginning of the transition in the $\mathrm{Tl}$ containing glasses did not show a linear correlation as seen in the glasses containing tin.

In an attempt to look at the origin of the transition, some representative samples were recovered after the pressurization cycle and their XRD was taken as shown in Fig. 3. The XRD clearly shows that the samples remain amorphous when they are decompressed after undergoing a high pressure transition to the near metallic state. The semiconducting to near metallic transition may be taking place either due to crystallization as the pressure is increased or because of the reduction in the energy gap due to the application of pressure. In the first case, the high pressure phase is crystalline and in the second case, the high pressure phase is likely to be amorphous. The behavior of the samples when the pressure was reduced from $9.5 \mathrm{GPa}$ to atmospheric pressure was probed and representative curves are shown in Fig. 4. These clearly indicate that the semiconductor to near metallic transition is exactly reversible with the removal of the pressure. The chalcogenide glasses $\mathrm{Ge}-\mathrm{Te}$ [15] and Ge-Tl-Se [21] have also shown a discontinuous glassy semiconductor to crystalline transition under the application of pressure; however, they do not revert back to the amorphous phase on removal of pressure but continue to remain in the crystalline phase. The fact that the samples in the present study remain amorphous even after undergoing a compression decompression cycle supports the idea that they remain amorphous in the high pressure phase, too, indicating that the transition is due to the reduction in energy gap under high pressure rather than a phase change from amorphous to crystalline. Xray crystallography of the samples under high pressure may be needed to confirm the phase of the sample at high pressure.

Electrical switching in glassy chalcogenides can occur in two different ways and are classified as memory type switching and threshold type switching. In the case of memory switching, the sample switches from a high resistance amorphous phase to a low resistance crystalline phase on the application of increasing electric field. On the removal of the field, the sample continues to remain in the low resistance crystalline phase. In the case of threshold switching, the sample goes from high resistance to low resistance on the application of an electric field and then returns to the high resistance state on the removal of the field i.e. the switching is reversible. The Ge-Te-Sn samples undergo memory type of switching [27] which indicates that amorphous to crystalline phase transition takes place easily in these samples. However, the semiconductor to near metallic transition observed under the application of pressure seems to be reversible with its origins in the reduction of the energy bandgap rather than a structural phase change.

As mentioned before, chalcogenide glasses have been observed to show evidence of percolation thresholds [2931 as a function of composition or increasing coordination number. The present $\mathrm{Ge}-\mathrm{Te}-\mathrm{Sn}$ samples do not exhibit this in their switching behavior. A plot of the normalized resistivity as a function of Sn content at a constant pressure of $1.3 \mathrm{GPa}$, shown in Fig. 5, also does not reveal the presence of percolation thresholds and is in agreement with the switching studies.

\section{Conclusions}

Bulk Ge-Te-Sn glasses are found to exhibit a sudden transition in their normalized electrical resistivity as a function of pressure undergoing a semiconductor to near metallic transition at pressures around 1.5-2.5 GPa. The transition pressure decreases with increasing tin content due to the increased metallicity of Sn compared to Te. XRD studies indicate that the samples remain amorphous even after a compression decompression cycle. The transition is exactly reversible, and provides an indication that it may be arising due to a reduction in the band gap due to pressure. 


\section{Acknowledgments}

K.N.N. Prasad is grateful to Srividya of IISc for her valuable help and management of BNM Institute of Technology for their support and encouragement.

\section{References}

[1] A.V. Kolobov, J. Tominaga, J. Mater. Sci. Mater. Electron. 14, 677 (2003).

[2] L. Greer, N. Mathur, Nature 437, 1246 (2005).

[3] H. Lee, Y.K. Kim, D. Kim, D.H. Kang, IEEE Trans. Magn. 41, 1034 (2005).

[4] J.Ch. Rhee, M. Okuda, T. Matsushita, Jpn. J. Appl. Phys. 26, 102 (1987).

[5] T. Nagano, E. Ueda, Sh. Onayama, R. Katayama, Hi. Hamada, Y. Ono, Jpn. J. Appl. Phys. 32, 5263 (1993).

[6] K. Uchino, K. Takada, T. Ohno, H. Yoshida, Y. Kobayashi, Jpn. J. Appl. Phys. 32, 5354 (1993).

[7] J. Biceramo, S.R. Ovshinsky, J. Non-Cryst. Solids 74, 75 (1985).

[8] S. Murugavel, K.V. Acharya, S. Asokan, J. Noncryst. Solids 191, 327 (1995).

[9] S. Asokan, G. Parthasarathy, E.S.R. Gopal, Philos. Mag. B 57, 49 (1988).

[10] G. Parthasarathy, E.S.R. Gopal, Bull. Mater. Sci. 7, 271 (1985).

[11] G. Parthasarathy, S. Asokan, S.S.K. Titus, R.R. Krishna, Phys. Lett. A 131, 441 (1988).

[12] G. Parthasarathy, R. Ramakrishna, S. Asokan, E.S.R. Gopal, J. Mater. Sci. Lett. 58, 809 (1986).

[13] S.S.K. Titus, S. Asokan, R. Ramakrishna, E.S.R. Gopal, Philos. Mag. B 62, 553 (1990).

[14] S. Asokan, E.S.R. Gopal, G. Parthasarathy, J. Mater. Sci. 21, 625 (1986).
[15] G. Parthasarathy, A.K. Bandyopadhyay, S. Asokan, E.S.R. Gopal, Solid State Commun. 51, 195 (1984).

[16] S.S.K. Titus, S. Asokan, E.S.R. Gopal, High Press. Res. 10, 629 (1992).

[17] D. Verma, B.H. Sharmila, K. Rukmani, S. Asokan, High Press. Res. 28, 55 (2008).

[18] B.H. Sharmila, J.T. Devaraju, S. Asokan, J. NonCryst. Solids 303, 372 (2002).

[19] D.P. Gosain, K.L. Bhatia, G. Parthasarathy, E.S.R. Gopal, Phys. Rev. B 32, 2727 (1985).

[20] S.K. Minomura, K. Aoki, O. Shimomura, Electronic Phenomena in Non-Crystalline Solids, Nauka, Leningrad 1976.

[21] G.M. Naik, G. Parthasarathy, E.S.R. Gopal, P.S. Narayanan, J. Mater. Sci. Lett. 4, 1017 (1985).

[22] K.N.N. Prasad, M.M. Rahman, K. Rukmani, S. Asokan, High Press. Res. Int. J. 34, 309 (2014).

[23] C.C. Bradley, High Pressure Methods in Solid State Research, Butterworths, London 1969.

[24] A.K. Bandyopadhyay, A.V. Nalini, E.S.R. Gopal, S.V. Subramanyam, Rev. Sci. Instrum. 51, 136 (1980).

[25] W. Jr. Klement, A. Jayaraman, G.C. Kennedy, Phys. Rev. 131, 632 (1963).

[26] M. Xu, Y.Q. Cheng, L. Wang, H.W. Sheng, Y. Meng, W.G. Yang, X.D. Han, E. Ma, Proc. Natl. Acad. Sci. USA 109, E1055 (2012).

[27] C. Das, G. Mohan Rao, S. Asokan, J. Non-Cryst. Solids 358, 224 (2012).

[28] J.M. Marshall, A.E. Owen, Philos. Mag. 22, 903 (1971).

[29] J.C. Phillips, M.F. Thorpe, Solid State Commun. 53, 699 (1985).

[30] J.C. Phillips, Phys. Rev. B 31, 8157 (1985).

[31] M.F. Thorpe, J. Non-Cryst. Solids 57, 355 (1983). 